This is a author version of

D. Schlipf, L. Y. Pao, and P. W. Cheng, "Comparison of feedforward and model predictive control of wind turbines using LIDAR," published in Proceedings of the Conference on Decision and Control, Maui, USA, 2012.

http://ieeexplore.ieee.org/xpls/abs_all.jsp?arnumber $=6426063$

\title{
Comparison of Feedforward and Model Predictive Control of Wind Turbines Using LIDAR
}

\author{
David Schlipf ${ }^{1}$, Lucy Y. Pao ${ }^{2}$ and Po Wen Cheng ${ }^{1}$
}

\begin{abstract}
LIDAR systems are able to provide preview information of wind disturbances at various distances in front of wind turbines. This technology paves the way for new control concepts such as feedforward control and model predictive control. This paper compares a nonlinear model predictive controller and a feedforward controller to a baseline controller. Realistic wind "measurements" are obtained using a detailed simulation of a LIDAR system. A full lifetime comparison shows the advantages of using the wind predictions to reduce wind turbine fatigue loads on the tower and blades as well as to limit the blade pitch rates. The results illustrate that the feedforward controller can be combined with a tower feedback controller to yield similar load reductions as the model predictive controller.
\end{abstract}

\section{INTRODUCTION}

An important design goal for large wind turbines is to reduce fatigue and extreme loads of the structure by tuning the control. This is a challenging task because transient events such as gusts represent an unknown disturbance to the control system. Conventional feedback controllers can only provide delayed compensation for such excitations, since the disturbance effects must propagate through the entire wind turbine before showing its effects in the measured outputs. This usually results in additional loads for the wind turbine and requires high actuator rates for the disturbance compensation. Those effects can be avoided, if the wind ahead of the wind turbine is measured by remote sensing techniques such as LIDAR (LIght Detection And Ranging) and the information is fed to the turbine controller.

While early work on LIDAR-assisted control was reported in [1], this field of investigation has increased significantly in recent years, and several feedforward and model predictive controllers have been proposed for load reduction or increasing the energy yield, see e.g., [2]-[8]. The present work illustrates that a good understanding of the relationship between meteorology, LIDAR technology, and system dynamics can lead to the design of a simple feedforward controller that can compete with a nonlinear model predictive controller (NMPC).

This paper is organized as follows. Section II summarizes the modeling of the wind turbine. Section III describes the LIDAR sensing process, and the different controllers are outlined in Section IV. Section V presents simulation results, and conclusions and future work are discussed in Section VI.

${ }^{1}$ D. Schlipf and P.W. Cheng are with Stuttgart Wind Energy, Institute of Aircraft Design, University of Stuttgart, Germany schlipf and cheng at ifb.uni-stuttgart.de

${ }^{2}$ L.Y. Pao is with the Department of Electrical, Computer, and Energy Engineering, University of Colorado, Boulder, USA pao at colorado.edu

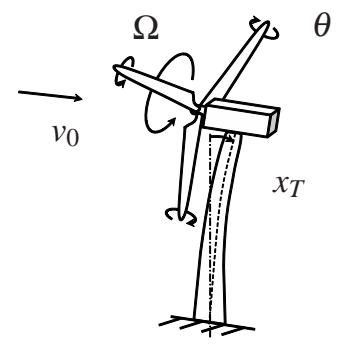

Fig. 1. Degrees of freedom for the reduced nonlinear model.

\section{Modeling OF The Wind Turbine}

The crucial part of a successful feedforward and model predictive controller design is the adequate modeling of the dynamic system to be controlled. The model should be simple enough to allow a partial system inversion (for the feedforward controller design) and simulations in reasonable computation time (for the NMPC), and at the same time it should be accurate enough to capture the system dynamics that are relevant for wind turbine control.

Classically aeroelastic simulation environments for wind turbines, such as FAST [9] (used later in this work), provide models close to reality but far too complex to be used for controller design. In addition, current remote sensing methods such as LIDAR are not able to provide a wind field estimate with comparable details to a generic wind field used by aeroelastic simulations (generated in this work with TurbSim [10]). In this section, a turbine model with three degrees of freedom (see Figure 1) is derived from physical fundamentals, and the wind field is reduced to the rotor effective wind speed which is measurable with existing LIDAR technology.

The first tower fore-aft bending mode, the rotational motion, and the collective pitch actuator are based on [11]:

$$
\begin{aligned}
J \dot{\Omega}+M_{g} / i_{g b} & =M_{a}\left(\dot{x}_{T}, \Omega, \theta, v_{0}\right) \\
m_{T e} \ddot{x}_{T}+c_{T} \dot{x}_{T}+k_{T} x_{T} & =F_{a}\left(\dot{x}_{T}, \Omega, \theta, v_{0}\right) \\
\ddot{\theta}+2 \xi \omega \dot{\theta}+\omega^{2}\left(\theta-\theta_{c}\right) & =0 .
\end{aligned}
$$

Equation (1a) models the drive-train dynamics, where $\Omega$ is the rotor speed, $M_{a}$ is the aerodynamic torque, $M_{g}$ is the electrical generator torque, $x_{T}$ is the tower top fore-aft displacement, $\theta$ is the effective collective blade pitch angle, and $v_{0}$ is the rotor effective wind speed. Moreover, $i_{g b}$ is the gear box ratio, and $J$ is the overall sum of the moments of inertia about the rotation axis. Equation (1b) describes the tower fore-aft dynamics, where $F_{a}$ is the aerodynamic thrust, and $m_{T e}, c_{T}$, and $k_{T}$ are the tower equivalent modal 


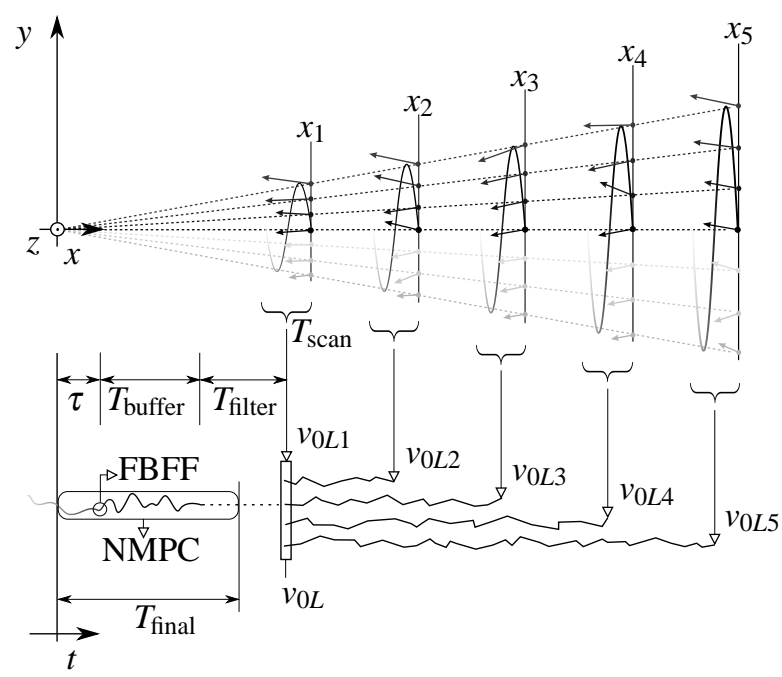

Fig. 2. Scope of the wind prediction: The line-of-sight wind speeds are measured at fixed distances, corrected, averaged over the last trajectory, time shifted, and combined to form a preview of the rotor effective wind speed.

mass, structural damping, and bending stiffness, respectively. Finally, equation (1c) is a second-order model of the blade pitch actuator, where $\omega$ is the undamped natural frequency, $\xi$ is the damping factor of the pitch actuator, and $\theta_{c}$ is the collective blade pitch control input.

The nonlinearity in the reduced model resides in the aerodynamic torque and thrust acting on the rotor with radius $R$ :

$$
\begin{aligned}
M_{a} & =\frac{1}{2} \rho \pi R^{3} \frac{c_{P}(\lambda, \theta)}{\lambda} v_{r e l}^{2} \\
F_{a} & =\frac{1}{2} \rho \pi R^{2} c_{T}(\lambda, \theta) v_{r e l}^{2},
\end{aligned}
$$

where $\rho$ is the air density, $\lambda$ is the tip-speed ratio

$$
\lambda=\frac{\Omega R}{v_{\text {rel }}},
$$

and $c_{P}$ and $c_{T}$ are the effective power and thrust coefficients, respectively. The nonlinear $c_{P}$ and $c_{T}$ coefficients can be obtained from steady-state simulation. The relative wind speed $v_{r e l}$ is defined as a superposition of tower top speed and mean wind speed

$$
v_{r e l}=\left(v_{0}-\dot{x}_{T}\right)
$$

and is used to model the aerodynamic damping.

\section{Simulated LiDAR Measurements}

LIDAR assisted controllers need a preview of the rotor effective wind speed, which has to be extracted from threedimensional wind fields. For better understanding of the preview provided by a real LIDAR system and to give an outlook of necessary algorithms needed in real applications, the scanning of the wind fields during the aeroelastic simulations with a detailed LIDAR simulator and the reconstruction of the rotor effective wind speed are presented in this section.
In this work, a pulsed system with a circular trajectory is used, which is performed within $T_{\text {scan }}=2.4 \mathrm{~s}$ with 12 focus points in 5 focus distances equally distributed between $x_{1}=63 \mathrm{~m}$ and $x_{5}=189 \mathrm{~m}$ (see Figure 2). This trajectory was realized by a real scanning LIDAR system installed on the nacelle of a $5 \mathrm{MW}$ turbine (see [12]). Effects from the experiment, such as collision of the laser beam with the blades and mechanical constraints of the scanner, are considered to obtain realistic simulations.

Taylor's frozen turbulence hypothesis assumes that the turbulent wind field moves with the average wind speed and is unaffected when approaching the rotor. This hypothesis is used in the simulation of the measurements and for the wind speed estimation. To account for the volume measurement of the LIDAR system, the wind field is not only analyzed at the focus points, but also in the area around the focus point along the laser beam. Here, a Gaussian shape weighting function $f_{L}(a)$ is used, depending on the distance $a$ to the focus point with full width at half maximum (FWHM) of $W=30 \mathrm{~m}$, following the considerations of [13]. It is possible to calculate the line-of-sight wind speed of each focus point with the LIDAR weighting function $f_{L}(a)$ by

$$
v_{l o s}=\int_{-\infty}^{\infty}\left(l_{x} u(a)+l_{y} v(a)+l_{z} w(a)\right) f_{L}(a) d a,
$$

where $\left[\begin{array}{lll}l_{x} & l_{y} & l_{z}\end{array}\right]^{T}$ is the normalized laser beam vector and $\left[\begin{array}{lll}u(a) & v(a) & w(a)\end{array}\right]^{T}$ is the wind vector at a distance $a$ to the focus point.

For each distance $j$, the longitudinal wind component is reconstructed assuming lateral and vertical wind components to be zero and by averaging over the last trajectory

$$
v_{0 L j}(t)=\frac{1}{12} \sum_{i=1}^{12} v_{l o s, i j} / l_{x i} .
$$

The obtained time series $v_{0 L j}$ is time-shifted according to Taylor's frozen turbulence hypothesis (see Figure 2). The farthest right point of each line represents the newest measurement of the corresponding focus distance $x_{j}$, and the time to reach the first focus distance is assumed to be $\left(x_{j}-x_{1}\right) / \bar{v}$, where $\bar{v}$ is the mean wind speed. The LIDAR estimate of the rotor effective wind speed $v_{0 L}(t)$ is then calculated by

$$
v_{0 L}(t)=\frac{1}{5} \sum_{j=1}^{5} v_{0 L j}\left(t-\left(x_{j}-x_{1}\right) / \bar{v}\right) .
$$

If the requested preview is larger than $x_{1} / \bar{v}$, the average is made only over the last four distances and so on. The wind speed preview $v_{0 L}$ is filtered by a low-pass filter, and the time delay introduced by the filter has to be considered as explained in the next section.

\section{Controller Design}

In this section, the feedforward controller and the NMPC using the wind speed preview information are derived. The baseline controller (FB) is based on feedback only and is implemented as described in [14]. It combines a variable speed generator torque controller and a collective pitch controller. 


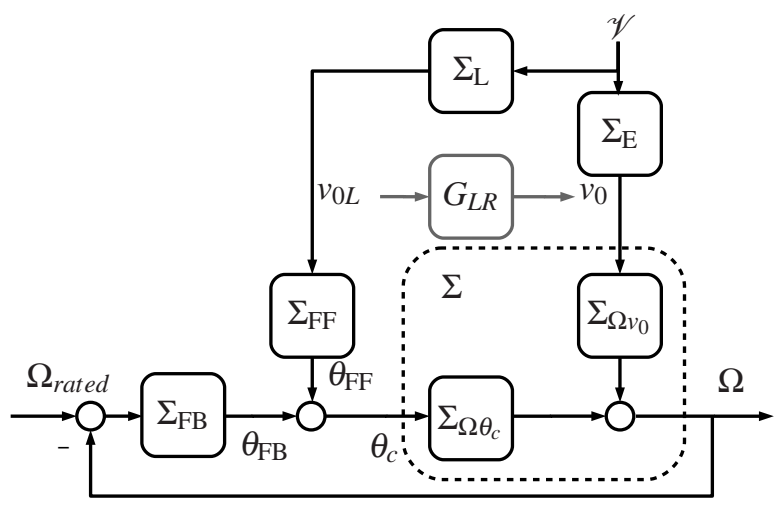

Fig. 3. Feedforward control: The feedforward controller $\Sigma_{\mathrm{FF}}$ tries to compensate for the effects of the wind field $\mathscr{V}$ to the rotor speed $\Omega$.

\section{A. The Feedforward Controller}

The feedforward controller (FBFF, see Figure 3) is based on the work in [4] and combines the baseline feedback controller with a feedforward update. The main control goal of the collective pitch feedback controller $\Sigma_{\mathrm{FB}}$ is to maintain the rated rotor speed $\Omega_{\text {rated }}$ by adjusting the pitch angle $\theta_{c}$. A wind field $\mathscr{V}$ evolves to $v_{0}$ on its way to the turbine and disturbs the system $\Sigma$. The measurement of the wind field in front of the turbine by a LIDAR system $\Sigma_{\mathrm{L}}$ yields $v_{0 L}$. The disturbance could be perfectly compensated by a feedforward controller

$$
\Sigma_{\mathrm{FF}}=-\Sigma_{\Omega \theta_{c}}^{-1} \Sigma_{\Omega v_{0}} \Sigma_{\mathrm{E}} \Sigma_{\mathrm{L}}^{-1},
$$

if the complete system is known and $\Sigma_{\Omega \theta_{c}}$ as well as $\Sigma_{\mathrm{L}}$ are invertible. Due to its complexity, the inversion of $\Sigma_{\Omega \theta_{c}}$ cannot be found for an aeroelastic model, but is computable for the reduced nonlinear model presented above. With the stationary pitch curve $\theta_{s s}\left(v_{0 s s}\right)$, which can be obtained from simulations or measurements, the part $-\Sigma_{\Omega \theta_{c}}^{-1} \Sigma_{\Omega v_{0}}$ is:

$$
\theta_{\mathrm{FF}}=\theta_{s s}\left(\left(\ddot{v}_{0}+2 \xi \omega \dot{v}_{0}+\omega^{2} v_{0}\right) / \omega^{2}\right) .
$$

In reality, $v_{0}$ cannot be measured perfectly due to the limitation of the LIDAR system, and $\Sigma_{\mathrm{E}}$ is quite complex to model. However, the transfer function $G_{L R}$ from the LIDAR estimate of the wind speed to the rotor effective wind speed can be analytically modeled or estimated from simulated or measured data via the auto correlation spectrum of the measured wind speed $S_{L L}$ and the cross correlation spectrum $S_{S L}$ between the measured and the rotor effective wind speed:

$$
G_{L R}=\frac{S_{L R}}{S_{L L}}
$$

Figure 4 shows $\left|G_{L R}\right|$ for a $1 \mathrm{~h}$ simulation with a full aeroelastic model and a turbulent wind field (mean wind speed $\bar{v}=16 \mathrm{~m} / \mathrm{s}$, turbulence intensity of $18 \%$ ). The rotor effective wind speed $v_{0}$ is obtained from simulated turbine data by an estimator using (1a) and (2a) similar to the one presented in [15] and $v_{0 L}$ from the LIDAR simulator presented above. Because of the Kaimal wind spectra used for these simulations and the LIDAR weighting function with fixed length, the shape of $\left|G_{L R}\right|$ for different $\bar{v}$ will

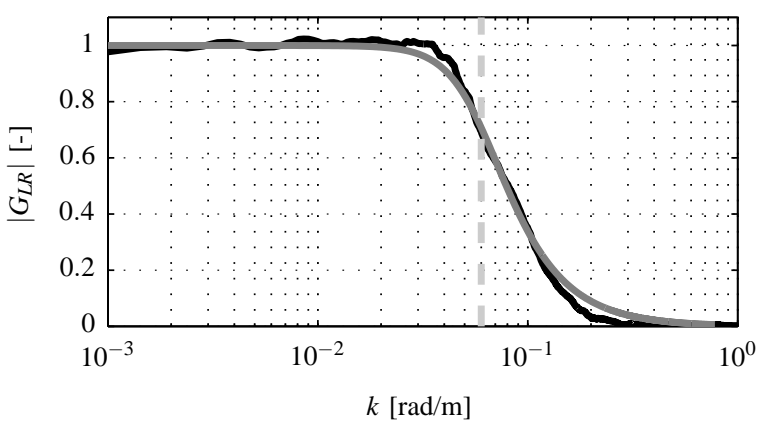

Fig. 4. Black: Magnitude of the transfer function $G_{L R}$ for a $1 \mathrm{~h}$ simulation. Dark gray: Fitted filter. Light gray: maximum wavenumber $\hat{k}$.

be similar over $k=2 \pi f / \bar{v}$, but different over $f$. Due to its low pass behaviour and the preview provided by the LIDAR, the transfer function is approximated by a secondorder Butterworth filter $G_{\text {filter }}$ and a time delay:

$$
G_{L R} \approx G_{\text {filter }}(s) e^{T_{\text {buffer }} s} .
$$

The time delay is obtained from the following considerations: With Taylor's hypothesis, the wind needs the time $x_{1} / \overline{v_{1}}$ to travel from the first focus distance to the turbine. Due to the average over the full trajectory, $v_{0 L}$ is already delayed by $T_{\text {scan }} / 2$. The filter delay is approximated by $T_{\text {filter. }}$. For using the filtered wind in the feedforward controller (9) instead of $v_{0}$, the signal has to be synchronized with $v_{0}$ reaching the rotor plane. Therefore the necessary time delay is

$$
T_{\text {buffer }}=\frac{x_{1}}{\bar{v}}-\frac{1}{2} T_{\text {scan }}-T_{\text {filter }}-\tau .
$$

The time $\tau$ compensates for small errors in the model reduction (see [3]).

The same $1 \mathrm{~h}$ simulation is repeated with different $\hat{k}$ and $\tau$ (see Figure 5) to evaluate if the feedforward controller yields the best performance with the proposed filter and time delay. The minimum in the standard deviation $\sigma(\Omega)$ of the rotor speed is found at $\hat{k}=0.06 \mathrm{rad} / \mathrm{m}$ and $\tau=0.4 \mathrm{~s}$, confirming the filter and time delay design.

In addition to the LIDAR assisted feedforward controller, a tower feedback controller is also added according to [16] to estimate the load reduction which can be achieved by measuring the tower acceleration $\ddot{x}_{T}$ and feeding the signal to the control system.

\section{B. The Nonlinear Model Predictive Controller}

The wind speed preview provided by LIDAR can also be used by a Nonlinear Model Predictive Controller. The controller presented in this section is designed to evaluate the performance of the aforementioned feedforward controller and is based on [5].

NMPC is an advanced control tool which predicts the future behavior of a system using a nonlinear internal model and the current measurements. With this information, the control actions necessary to regulate the plant are computed by solving an optimal control problem over a given time horizon. Part of the solution trajectory for the control inputs is transferred to the system, new measurements are gathered, 


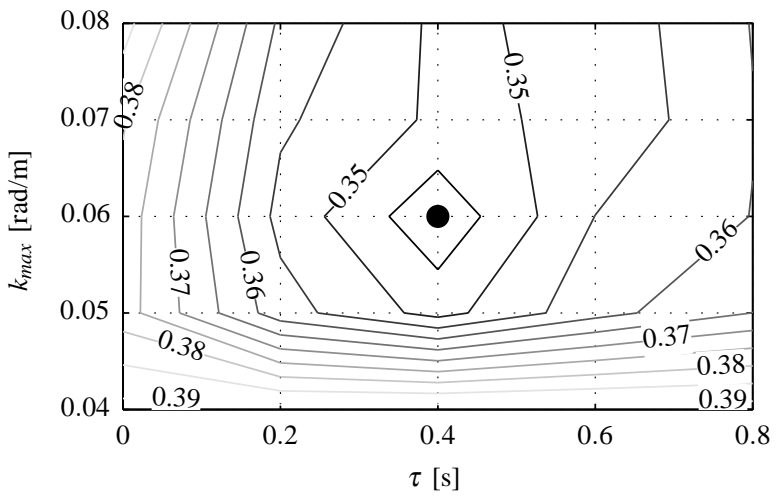

Fig. 5. Standard deviation of the rotor speed using the feedforward controller with different $\tau$ and $\hat{k}$ normalized by the standard deviation of the rotor speed when using the baseline controller.

and the optimal control problem is solved again at the next time step. Feedback is incorporated, since the current state of the turbine is implemented as the initial condition of the optimal control problem [17] at the next time step.

Here, the NMPC controls the collective pitch and is only active if the wind preview over the entire horizon is above $v_{\text {rated }}=11.2 \mathrm{~m} / \mathrm{s}$. If one value of the preview falls below this value, the baseline pitch controller is initialized with the current pitch angle and activated.

The considered optimal wind turbine control problem can be described as follows. The objective is to find the optimal control trajectory $u(\cdot)$ minimizing the cost functional $J_{O C P}$, which is defined as the integral over the time horizon $T_{\text {final }}$ of the objective functional $F$ from the actual time $t_{0}$ to the final time $t_{0}+T_{\text {final }}$, with the reduced nonlinear model and the set of constraints $H$. The crux of designing the NMPC is to translate the verbal formulation of the control goal to a mathematical formulation of $F$ and $H$. The optimal control goal can be stated as "minimizing the damage equivalent loads above rated wind speed without decreasing the energy production". In classic wind turbine control [18], this is in general done by limiting rotor speed and power above the rated wind speed.

The objective functional should be quadratic for computational reasons. This implies the weights to be independent of the system states and inputs, but they are allowed to be dependent on external disturbances. Here, $F$ is chosen to

$$
\begin{aligned}
F & =\quad Q_{1}\left(\Omega(\tau)-\Omega_{\text {rated }}\right)^{2} \\
& +Q_{2} \dot{x}_{T}^{2}(\tau) \\
& +R_{1}\left(v_{0}(\tau)\right) \dot{\theta}^{2}(\tau) .
\end{aligned}
$$

The first line of (13) penalizes the deviation from the rated rotor speed, and in the second line of (13) the tower foreaft velocity is penalized to minimize loads on the tower. The weight for the pitch rate $R_{1}\left(v_{0}(\tau)\right)$ is designed to penalize the pitch actuator rate. To account for the higher sensitivity of the pitch at higher wind speed, the static pitch angle over static wind speed $\theta_{s s}\left(v_{s s}\right)$ is used together with the gain correction factor $G K(\theta)$ from [14] and the static weight $R_{1}$ :

$$
R_{1}\left(v_{0}(\tau)\right)=R_{1} / G K\left(\theta_{s s}\left(v_{0}(\tau)\right)\right)
$$

The set of constraints $H$, which can be organized in the form $H \geq 0$, is chosen as

$$
\begin{aligned}
\Omega(\tau) & \leq 1.2 \Omega_{\text {rated }} \\
\theta_{\text {min }} \leq \theta(\tau) & \leq \theta_{\text {max }} \\
|\dot{\theta}(\tau)| & \leq \dot{\theta}_{\text {max }}
\end{aligned}
$$

The constraint (15a) limits the rotor speed to $120 \%$ of $\Omega_{\text {rated }}$, (15b) limits the pitch angle to its feasible positions, and (15c) constrains the pitch rate.

The optimal control problem is converted by the Direct Multiple Shooting method [17] into a nonlinear program. Here, the control inputs are discretized in $K$ piecewise constant stages. The ODEs of the model are solved numerically on each interval. The optimization is performed over the set of initial values for all states and the control outputs. Additional constraints are applied to ensure that the states at the end of each stage coincide with the initial conditions of the subsequent stage. This method gives significant improvements over the Direct Single Shooting approach, especially with respect to numerical stability.

The nonlinear program can be solved iteratively with Sequential Quadratic Programming (SQP). The separation of the optimization problem into multiple stages results in a faster solution. This is due to the better approximation of the Lagrangian Hessians of the nonlinear problem parts in each stage by low rank updates [19].

Here, Omuses [19] is used as a front-end to the large-scale SQP-type nonlinear optimization solver HQP. The prediction horizon is set to $T_{\text {final }}=5.6 \mathrm{~s}$ as the minimal preview time of the LIDAR (see [5]). The time steps are set equal to the LIDAR update rate, resulting in $K=T_{\text {final }} /\left(T_{\text {scan }} / 12\right)=28$ stages. The differential equations are solved with a fourthorder explicit Runge Kutta method.

To avoid resonance cases, notch filters (Butterworth, 2nd order) with stop band at $\left[0.9 f_{3 P}, 1.1 f_{3 P}\right]$ and $\left[0.9 f_{0}, 1.1 f_{0}\right]$ are used, where $f_{0}$ is the natural frequency of the tower, and $f_{3 P}$ is the frequency at which the blades pass by the tower. The number of control steps applied in a feedforward control to the system after each optimization is chosen as $K_{\mathrm{FF}}=3$ to run in real time on a typical current PC. This implies that the optimization is repeated with new measurements each $0.6 \mathrm{~s}$ to close the control loop. The proof of closed-loop stability of a nonlinear and constrained system solved by a model predictive controller is beyond the scope of this work and is fairly complex as $J_{O C P}$ has to be a local Lyapunov function. There are some theoretical approaches [20] and practical recommendations [21], but the following results will show that there is no evidence of any stability problem in this case.

The NMPC controller needs the full-state vector at the start of the optimization horizon. Only the rotor speed $\Omega$, the tower fore-aft-acceleration $\ddot{x}_{T}$, the pitch angle $\theta$, and the pitch rate $\dot{\theta}$ can be considered as measurable signals. Therefore, an estimator is implemented to reconstruct $\dot{x}_{T}$ and $x_{T}$, combining a static nonlinear estimation of the aerodynamic thrust and a linear Luenberger estimator [5]. 


\section{Considerations for Real Applications}

The presented feedforward controller has the advantage that it can be easily integrated in state-of-the-art control systems. For real applications, it is beneficial to use a pitch rate update $\dot{\theta}_{\mathrm{FF}}$ instead of $\theta_{\mathrm{FF}}$, see [4]. This allows a gradual application of the feedforward controller. The maximum wavenumber $\hat{k}$ can be estimated online from LIDAR and turbine data. There are two main issues which have to be considered for an implementation of the presented NMPC. An intermediary result can far away from the optimum due to the Direct Multiple Shooting method, and the presented approach leads to the iterative solution of a non-convex optimization problem and thus there is no guarantee to find the global minimum in the allotted time slot. However, these restrictions do not have a strong impact on the given implementation. The time needed to execute the optimization is recorded for all 33 simulations of Section V. Only $0.4 \%$ of the optimizations last longer than the allotted time.

\section{Simulation Results}

Simulations are performed with an aeroelastic model of a $5 \mathrm{MW}$ three-bladed pitch-controlled variable-speed wind turbine designed by the National Renewable Energy Laboratory (NREL) as described in [14]. It is supplemented with a second-order linear model to account for the pitch actuator dynamics, and the pitch rate limit is $8^{\circ} / \mathrm{s}$. There are a total of 34 dynamic states of the aeroelastic model.

To estimate the benefit for fatigue load reduction, various 10 min-simulations for each controller with a set of turbulent TurbSim wind fields are conducted, featuring Atype turbulence intensity according to IEC 61400-1 [22] and a Rayleigh distribution with $C=12 \mathrm{~m} / \mathrm{s}$. The baseline generator torque controller is enabled in all simulations. The integral parameter $T_{i}$ of the collective pitch feedback controller is modified to $T_{i, \mathrm{FBFF}}=3 T_{i}$, when used with the feedforward update, to lower the tower loads (see [4]). The NMPC coupled to the LIDAR simulator and the nonlinear estimator is tuned to have high load reduction on tower and blades together with low pitch activity.

Figure 6 shows a sample simulation with mean wind speed $22 \mathrm{~m} / \mathrm{s}$ and turbulence intensity $16 \%$. In the first plot, the rotor effective wind $v_{0}$ estimated from turbine data and the measurement $v_{0 L}$ from the LIDAR simulation are compared. In the second plot, the reduced pitch activity for all LIDAR assisted controllers can be observed compared to the baseline controller. The remaining plots show that with the NMPC and the feedforward controller, the variation in rotor speed and tower base fore-aft bending moment $M_{y T}$ can be reduced. The effect of the different controllers is more obvious in the frequency domain (see Figure 7). The NMPC and the feedforward controller can reduce the influence of the wind disturbance to rotor speed and to the tower base fore-aft bending moment for frequencies up to the $1 \mathrm{P}$ frequency at $0.2 \mathrm{~Hz}$. The pitch rate is also reduced in this region. The tower feedback controller, which is active in the FBFF case, is able to further reduce the loads at the natural frequency $f_{0}$ on the tower at the expense of higher pitch rates.

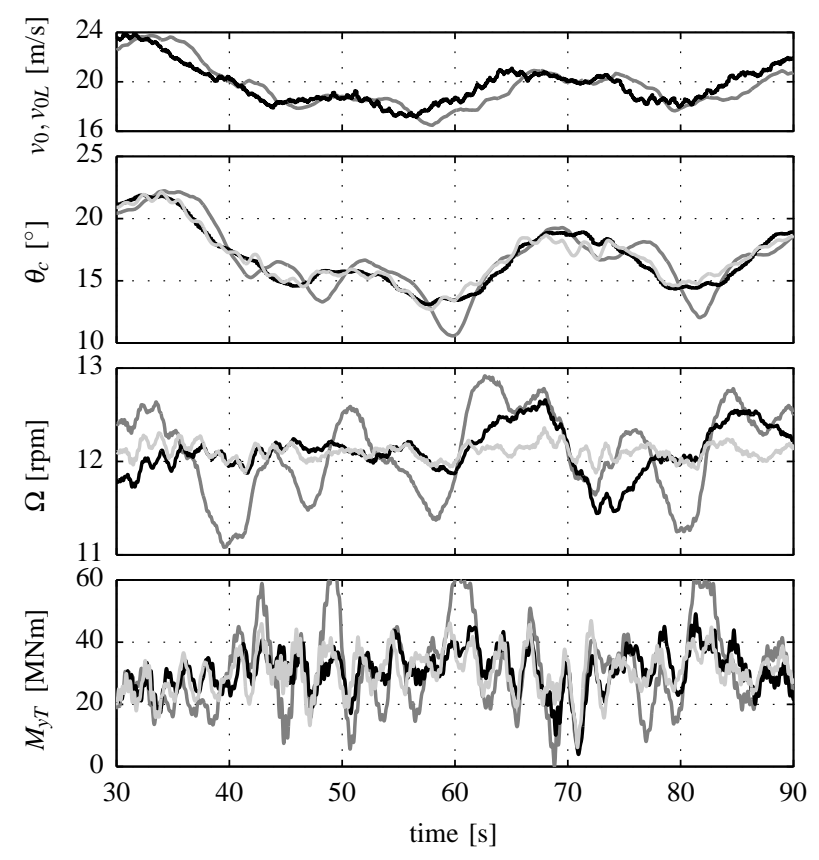

Fig. 6. Top: rotor effective wind speed from turbine (gray) and LIDAR (black). Below: pitch angle demand, rotor speed, and tower base fore-aft bending moment for FB (dark gray), NMPC (black), and FBFF (light gray).
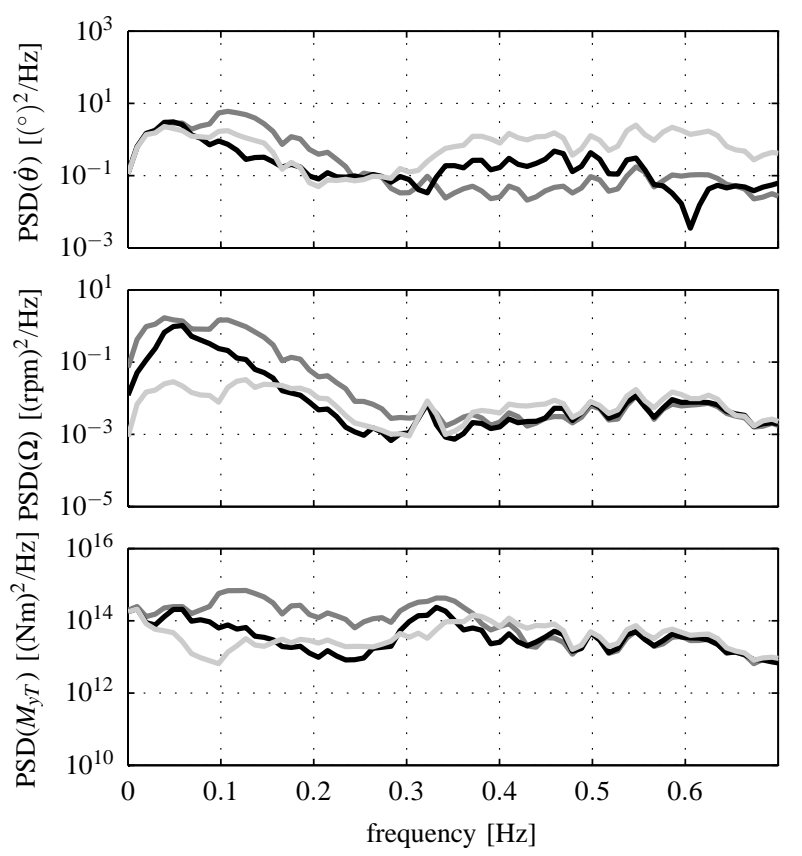

Fig. 7. Power spectral densities: FB (dark gray), NMPC (black) and FBFF (light gray).

TABLE I

OVERALL PERFORMANCE FOR THE DIFFERENT CONTROLLERS.

\begin{tabular}{l|rrrrrr} 
& $\begin{array}{r}M_{y T} \\
{[\mathrm{MNm}]}\end{array}$ & $\begin{array}{r}M_{\text {oop } 1} \\
{[\mathrm{MNm}]}\end{array}$ & $\begin{array}{r}M_{L S S} \\
{[\mathrm{MNm}]}\end{array}$ & $\begin{array}{r}\mathrm{EP} \\
{[\mathrm{GWh}]}\end{array}$ & $\begin{array}{r}\sigma(\dot{\theta}) \\
{[\%]}\end{array}$ & $\begin{array}{r}\sigma(\Omega) \\
{[\mathrm{rpm}]}\end{array}$ \\
\hline FB & 87.66 & 12.87 & 2.89 & 548.42 & 0.46 & 0.59 \\
NMPC & 72.58 & 11.62 & 2.81 & 549.38 & 0.36 & 0.55 \\
FBFF & 69.88 & 11.36 & 2.71 & 550.17 & 0.34 & 0.51
\end{tabular}



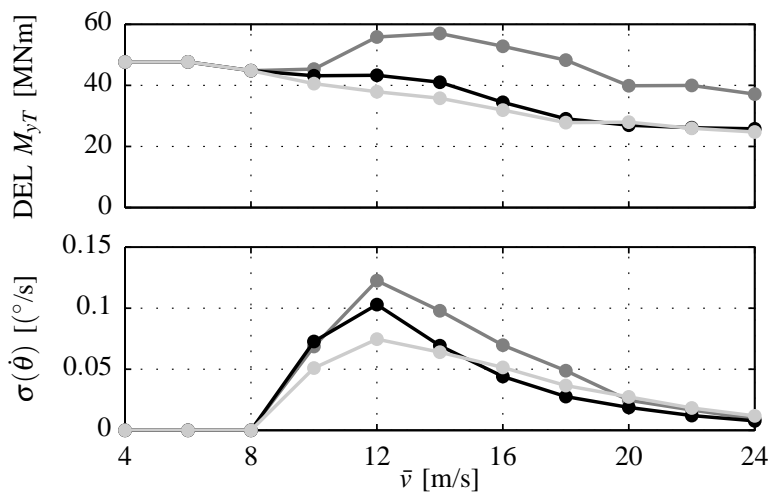

Fig. 8. Distribution of lifetime weighted DEL of the tower base fore-aft bending moment and the lifetime weighted standard deviation for pitch rate for FB (dark gray), NMPC (black), and FBFF (light gray).

Over all simulations, the NMPC and the feedforward controller lead to satisfying control performance. Damage equivalent loads (DEL) are calculated with Wöhler exponents of 4 and 10, typical for steel and composite material [16]. The distribution of the lifetime weighted DEL (20 years, reference number of cycles $2 \times 10^{6}$ ) of the tower base foreaft bending moment is shown in Figure 8 .

Table I summarizes the results for all 33 simulations. For the LIDAR assisted controllers, the possible reduction in DEL of the tower base fore-aft bending moment $M_{y T}$, the out-of-plane bending moment $M_{\text {oop } 1}$ of blade 1 , and the low-speed shaft torque $M_{L S S}$ can be estimated to be approximately $20 \%, 10 \%$, and $3-6 \%$, respectively. The standard deviation of the pitch rate and the rotor speed are decreased by $25 \%$ and $7-13 \%$, respectively. Furthermore, the energy production (EP) can be increased slightly.

\section{CONClusions And Future Work}

In this paper, we have compared two controllers that use LIDAR measurements of the wind inflow to a wind turbine. One of the controllers is a feedforward controller designed based on an understanding of meteorology, LIDAR technology, and system dynamics; while the other controller is a nonlinear model predictive controller designed from theoretical mathematical principles. The controllers were evaluated with simulated measurements of a LIDAR system on a complex stochastic wind field and compared to a baseline controller. Promising load reductions on the tower and blades as well as a reduction in the pitch activity were achieved by both controllers. While the NMPC approach provides a theoretical framework for designing an "optimal" controller given a set of assumptions and constraints, the feedforward controller is less computationally complex and hence provides implementation advantages while yielding similar performance increases over the baseline controller.

The feedforward controller is currently being field tested on two $660 \mathrm{~kW}$ wind turbines at NREL. In future work, we plan to apply the NMPC approach for more complex wind turbine control scenarios, such as the control of floating offshore wind turbines using wind and wave preview.

\section{ACKNOWLEDGMENT}

Part of this research is funded by the German Federal Ministry for the Environment, Nature Conservation and Nuclear Safety (BMU) in the framework of the joint research project "LIDAR II". Partial support from the US National Renewable Energy Laboratory (NREL) is also gratefully acknowledged.

\section{REFERENCES}

[1] M. Harris, M. Hand, and A. Writght, "Lidar for turbine control," Technical Report NREL/TP-500-39154, 2006.

[2] J. Laks, L. Y. Pao, A. Wright, N. Kelley, and B. Jonkman, "Blade pitch control with preview wind measurements," in Proceedings of the 48th AIAA Aerospace Sciences Meeting Including the New Horizons Forum and Aerospace Exposition, Orlando, USA, 2010.

[3] F. Dunne, D. Schlipf, L. Y. Pao, A. D. Wright, B. Jonkman, N. Kelley, and E. Simley, "Comparison of two independent lidar-based pitch control designs," in Proc. 50th AIAA Aerospace Sciences Meeting Including the New Horizons Forum and Aerospace Exposition, 2012.

[4] D. Schlipf, T. Fischer, C. E. Carcangiu, M. Rossetti, and E. Bossanyi, "Load analysis of look-ahead collective pitch control using LiDAR," in Proceedings of the German Wind Energy Conference DEWEK, Bremen, Germany, 2010.

[5] D. Schlipf, D. J. Schlipf, and M. Kühn, "Nonlinear model predictive control of wind turbines using LIDAR," Wind Energy, 2012.

[6] A. Körber and R. King, "Nonlinear model predictive control for wind turbines," in Proceedings of the European Wind Energy Association Annual event, Brussels, Belgium, 2011.

[7] L. C. Henriksen, "Model predictive control of wind turbines," Ph.D. dissertation, Technical University of Denmark, 2011.

[8] K. A. Kragh, M. H. Hansen, and T. Mikkelsen, "Precision and shortcomings of yaw error estimation using spinner-based light detection and ranging," Wind Energy, 2012.

[9] J. Jonkman and M. L. Buhl, "FAST user's guide," NREL, Tech. Rep. NREL/EL-500-38230, August 2005.

[10] B. J. Jonkman, "TurbSim user's guide," Technical Report NREL/TP500-46198, September 2009.

[11] C. L. Bottasso, A. Croce, B. Savini, W. Sirchi, and L. Trainelli, "Aeroservo-elastic modelling and control of wind turbines using finiteelement multibody procedures," Multibody System Dynamics, vol. 16, pp. 291-308, 2006.

[12] A. Rettenmeier, O. Bischoff, M. Hofsäß, D. Schlipf, J. J. Trujillo, and M. Kühn, "Wind field analyses using a nacelle-based lidar system," in European Wind Energy Conference, Warsaw, Poland, 2010.

[13] J. P. Cariou, "Pulsed lidars," in Remote Sensing for Wind Energy. Risøreport Ris $\phi-I-3184(E N)$. Roskilde, Denmark: A. Peña and C. B. Hasager, June 2011, pp. 65-81, risø-DTU.

[14] J. Jonkman, S. Butterfield, W. Musial, and G. Scott, "Definition of a 5-MW reference wind turbine for offshore system development," Technical Report NREL/TP-500-38060, 2009.

[15] E. van der Hooft and T. G. van Engelen, "Estimated wind speed feed forward control for wind turbine operation optimization," European Wind Energy Conference, London, vol. 1, p. 9, 2004.

[16] E. Bossanyi, B. Savini, M. Iribas, M. Hau, B. Fischer, D. Schlipf, T. van Engelen, M. Rossetti, and C. E. Carcangiu, "Advanced controller research for multi-MW wind turbines in the UpWind project," Wind Energy, vol. 15, no. 1, pp. 119-145, 2012.

[17] R. Findeisen, "Nonlinear model predictive control: A sampled-data feedback perspective," Ph.D. dissertation, Universität Stuttgart, 2005.

[18] T. Burton, N. Jenkins, D. Sharpe, and E. Bossanyi, Wind Energy Handbook. New York, USA: John Wiley \& Sons, 2001.

[19] R. Franke, "Omuses - a tool for the optimization of multistage systems and HQP a solver for sparse nonlinear optimization," TU Ilmenau, Tech. Rep., 1998.

[20] D. Q. Mayne, J. B. Rawlings, C. V. Rao, and P. O. M. Scokaert, "Constrained model predictive control: Stability and optimality," $\mathrm{Au}$ tomatica, vol. 36, pp. 789-814, 2000.

[21] H. Chen and F. Allgöwer, "A quasi-infinite horizon nonlinear model predictive control scheme with guaranteed stability," Automatica, vol. 34, pp. 1205-1217, 1998.

[22] IEC 61400-1 third edition 2005-08 Wind turbines - Part 1: Design requirements, IEC Std., Rev. third edition, 2005. 